storms, and show that ANP and metyrosine, which are approved for use in the clinic in other contexts, might be effective in preventing this complication. It is generally assumed that the production of cytokines and their role in the activation of immune cells contributes to the efficiency of antitumour immune responses ${ }^{14}$. To ensure that antitumour effects are not diminished, it will be necessary to proceed cautiously when testing whether targeting catecholamine synthesis can reduce cytokine storms in a clinical setting.
Stanley R. Riddell is at the Fred Hutchinson Cancer Research Center, Seattle, Washington 98109, USA.

e-mail: sriddell@fredhutch.org

1. Rosenberg, S. A. Nature 411, 380-384 (2001)

2. Gangadhar, T. C. \& Vonderheide, R. H. Nature Rev. Clin. Oncol. 11, 91-99 (2014).

3. Hay, K. A. et al. Blood 130, 2295-2306 (2017).

4. Staedtke, V. et al. Nature 564, 273-277 (2018).

5. Medzhitov, R. Nature 454, 428-435 (2008).

6. Roberts, N. J. et al. Sci. Transl. Med. 6, 249ra111 (2014).

7. Flierl, M. A. et al. Nature 449, 721-725 (2007).
8. Bergquist, J., Tarkowski, A., Ekman, R. \& Ewing, A. Proc. Natl Acad. Sci. USA 91, 12912-12916 (1994).

9. Chatenoud, L. et al. Transplantation 49, 697-702 (1990).

10.Sadelain, M., Riviere, I. \& Riddell, S. Nature $\mathbf{5 4 5}$, 423-431 (2017).

11.Ladetzki-Baehs, K. et al. Endocrinology 148, 332-336 (2007).

12. Norelli, M. et al. Nature Med. 24, 739-748 (2018). 13.Giavridis, T. et al. Nature Med. 24, 731-738 (2018) 14. Kammertoens, T. et al. Nature 545, 98-102 (2017).

The author declares competing financial interests: see go.nature.com/2aycsnd for details.

\section{Finches choose parent lookalikes as mates}

\section{A preference for mating with similar individuals can have a key role in speciation. Research on Darwin's finches suggests that individuals might use the likeness of their parents as a guide for choosing mates.}

\section{LEWIS G. SPURGIN \& TRACEY CHAPMAN}

$\mathrm{N}$ ew species form when groups of individuals in a population become reproductively isolated and can no longer mate with each other to produce living, healthy offspring. For decades, evolutionary biologists have sought to understand the links between an individual's choice of mate and reproductive isolation between populations National Academy of Sciences, Grant and Grant ${ }^{2}$ provide evidence suggesting that two species of Darwin's finch learn features of their parents early in life and use this knowledge to inform their choice of mate in adulthood, a process known as sexual imprinting. Their study raises fascinating questions about the roles of learning and genetics in mate choice, and how matings between similar individuals and species ${ }^{1}$. Writing in Proceedings of the

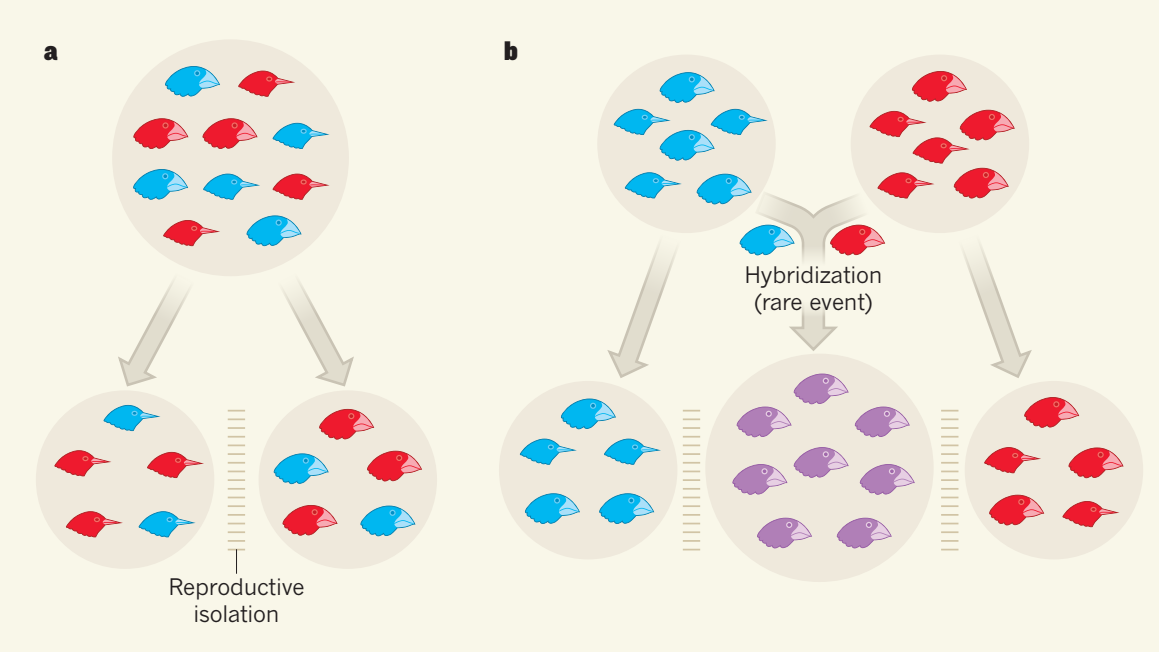

Figure 1 | Potential contributions of sexual imprinting to speciation. Grant and Grant ${ }^{2}$ report that, in Darwin's finches, sexual imprinting and assortative mating - learning parental features early in life and using this to choose a mate - can reduce the likelihood of dissimilar individuals mating with each other. Reproductive isolation caused by these and other factors might contribute to the evolution of new species in two ways. a, Speciation by fission involves the splitting of one species into two. In this example, imprinted mating preferences for beak size promote the formation of two new species with either small or large beaks. b, In speciation by fusion, a rare hybridization event between individuals of different species, followed by an imprinted preference to reproduce with similar individuals, promotes the formation of a new species alongside the two original ones. (assortative mating) drive the evolution of new species.

Darwin's finches live in the Galapagos archipelago. They are an iconic group of approximately 15 bird species that have contributed hugely to our understanding of natural selection and speciation ${ }^{3-5}$. Previous work has shown that the cultural inheritance of song can promote reproductive isolation between different species of Darwin's finch ${ }^{6}$. However, it was not known whether sexual imprinting based on morphological features such as body size and beak characteristics could similarly promote reproductive isolation, or play a part in the rare cases of mating between species that produce hybrid individuals.

If sexual imprinting is key in directing mate choice, then individuals should choose mates that resemble their parents, and also themselves. In addition, if sexual imprinting contributes to matings between species, then the parents of the hybrid individuals that result from such matings should more closely resemble the other species than their own. To test these hypotheses, Grant and Grant analysed 22 years of data on body size, beak size and beak shape in two finch species - Geospiza fortis and Geospiza scandens - living on the same island.

Grant and Grant found significant positive associations between certain features of the birds' chosen mates and those of their parents. For $G$. fortis, the body size of the chosen mate was strongly correlated with the body size of the chooser's father, but weakly correlated with that of the chooser's mother. The researchers did not explicitly test whether this imprinting was stronger in male or female offspring. For G. scandens, the beak length of male mates chosen by females was significantly associated with the beak shape and length of the female's father, although there were no other significant associations. Grant and Grant suggest that these imprinting patterns can promote assortative mating by body size and beak length in both species.

For matings between species, the results were less straightforward. In some cases, hybridizing individuals or their fathers from a given species were similar in size and shape to individuals from the other species. Although such results are intriguing, hybridization events are rare and sample sizes are small, so 
further research is needed to confirm whether they can be generalized to other species.

Grant and Grant frame their study as a test of sexual imprinting, but acknowledge that the correlations they observed could also arise if mating preferences were inherited genetically. There has been increasing support for the idea that sexual imprinting can reinforce reproductive isolation in birds and other species ${ }^{5}$. However, evidence for the existence of genetically inherited mating preferences in birds is limited. It is not yet clear whether learnt behaviour has a greater effect on mate choice than does genetic inheritance, or whether these inherited effects have been under-studied. Disentangling the roles of inherited and learnt mate preferences, and their consequences for speciation, is a key challenge for the future ${ }^{7}$.

The most powerful tests for identifying sexual imprinting use an experimental 'crossfostering' approach, in which offspring are swapped early in life and reared by unrelated individuals of the same or a different species ${ }^{8}$. There is also increasing interest in directly quantifying the genetic basis of mate choice using DNA sequencing 9 . We anticipate that future studies will combine experimental and genetic approaches to understand when and why learnt and inherited mating preferences evolve.

Grant and Grant's findings hint that sexual imprinting might have different effects in males and females and across different species. In a cross-fostering experiment in wild mice published last year, the strength of sexual imprinting differed substantially between two species ${ }^{8}$. Furthermore, in the mouse species in which imprinting was weaker, only males showed signs of imprinting. Why imprinting might be weaker and inherited preferences stronger in females of some species is not clear. This could occur if matings between species require more investment in time or effort from females than males, or if mate-choice patterns are influenced by differences in the extent of parental care or the social environment. The effects of inherited and learnt mate preferences are likely to be complex, and studies of a broad range of biological systems might be required to uncover their relative roles in nature.

The work by Grant and Grant links individual variation in mating preferences in Darwin's finches to the evolution of reproductive isolation, which is central to speciation. Sexual imprinting could have a role both in the 'classic' model of speciation, in which one species separates into two, and in the rarer process of speciation through hybridization, in which two different species mix to create a new one (Fig. 1). The possibility that Darwin's finches show sexual imprinting should encourage further experimental tests in other species to determine the role of imprinting in natural populations. Understanding how mating preferences evolve will shed light on the processes shaping past, present and future biodiversity.
Lewis G. Spurgin and Tracey Chapman are in the School of Biological Sciences, University of East Anglia, Norwich Research Park,

Norwich NR4 7TJ, UK.

e-mails:l.spurgin@uea.ac.uk;

tracey.chapman@uea.ac.uk

1. Irwin, D. E. \& Price, T. Heredity 82, 347-354 (1999).

2. Grant, P. R. \& Grant, B. R. Proc. Natl Acad. Sci. USA https://doi.org/10.1073/pnas.1813662115 (2018)

3. Grant, P. R. \& Grant, B. R. How and Why Species Multiply: The Radiation of Darwin's Finches (Princeton Univ. Press, 2011).
4. Lamichhaney, S. et al. Science 359, 224-228 (2018).

5. Verzijden, M. N. et al. Trends Ecol. Evol. 27, 511-519 (2012).

6. Grant, B. R. \& Grant, P. R. Evolution 50, 2471-2487 (1996).

7. Yeh, D. J., Boughman, J. W., Sætre, G.-P. \& Servedio, M. R. Evolution https://doi.org/10.1111/ evo.13500 (2018).

8. Delaney, E. K. \& Hoekstra, H. E. Evolution 72, 274-287 (2018)

9. Merrill, R. M. et al. Preprint at bioRxiv https://doi. org/10.1101/282301 (2018).

This article was published online on 26 November 2018.

\section{ORGANIC CHEMISTRY}

\section{An exciting tool for asymmetric synthesis}

A catalytic process driven by visible light converts a mixture of mirror-image isomers of compounds called allenes to a single mirror-image isomer - opening up avenues of research for synthetic chemistry. SEE LETTER P. 240

\section{CHENG YANG \& YOSHIHISA INOUE}

$\mathrm{M}$ olecules can exhibit a handedness, known as chirality. This is crucial to many aspects of chemistry and biology because the mirror-image isomers (enantiomers) of a chiral molecule can have distinctly different properties, reactivities and chemical or biological functions. For example, nature often uses just one enantiomer of a family of molecules as building blocks to construct sophisticated structures such as DNA, and in other biological processes. The development of methods for synthesizing chiral molecules asymmetrically - predominantly as a single enantiomer - is therefore one of the most important goals in organic and medicinal chemistry. On page 240, Hölzl-Hobmeier et al. ${ }^{1}$ report an approach that can also be used to achieve a seemingly impossible task in asymmetric synthesis: the light-induced, catalytic and apparently irreversible formation of single enantiomers of molecules called allenes from a one-to-one mixture of enantiomers (a racemic mixture).

One modern approach to asymmetric synthesis is to use light to induce the formation of a particular enantiomer of a molecule, a strategy called photochirogenesis. Often complementary to conventional methods of asymmetric synthesis, photochirogenesis is useful for making single enantiomers of molecules
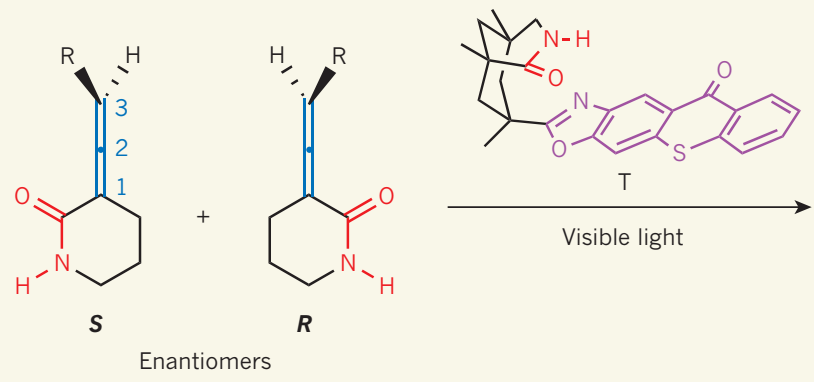

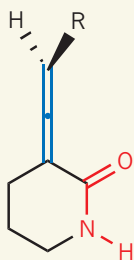

$\boldsymbol{R}$

Figure 1 | A light-activated deracemization process. In allene molecules, one carbon atom (designated $\mathrm{C} 2$ ) forms double bonds to its neighbouring carbon atoms ( $\mathrm{C} 1$ and $\mathrm{C} 3$; allene structure shown in blue). If two different groups are attached to each of $\mathrm{C} 1$ and $\mathrm{C} 3$, two mirror-image isomers (enantiomers) of the allene can form. Hölzl-Hobmeier et al. ${ }^{1}$ report a light-driven process known as a deracemization, in which a one-to-one mixture of allene enantiomers $(S$ and $R$ ) is converted into just the $R$-enantiomer. The reaction requires a catalyst called an enantiomeric photosensitizing template $(T)$, which contains a photosensitizer group (purple) that allows it to absorb visible light and transfer the energy to the allene. The motifs in red allow $T$ to form a complex with $S$ and $R$, as needed for the deracemization. R within the enantiomers represents various chemical groups; the solid wedge and the broken wedge represent bonds that project above and below the plane of the page, respectively. 